Bull. Austral. Math. Soc.

VOL. 74 (2006) [219-226]

\title{
ENSURING A FINITE GROUP IS SUPERSOLUBLE
}

\author{
R.A. BRYCE
}

A special case of the main result is the following. Let $G$ be a finite, non-supersoluble group in which from arbitrary subsets $X, Y$ of cardinality $n$ we can always find $x \in X$ and $y \in Y$ generating a supersoluble subgroup. Then the order of $G$ is bounded by a function of $n$. This result is a finite version of one line of development of B.H. Neumann's well-known and much generalised result of 1976 on infinite groups.

\section{BACKGROUND}

A group is Abelian, of course, if every pair of its elements generates an Abelian subgroup. In [10] Neumann generalised this showing that a group is centre-by-finite if in every infinite subset of it there is a pair of elements that generates an Abelian subgroup. This result has itself been generalised by many authors, although usually proving results that are vacuous in a finite group. The present article gives a finite version of some of the post-Neumann work.

Our motivation includes the following results. Firstly Lennox and Wiegold [7] proved, among other things, that a finitely generated soluble group is finite-by-nilpotent if and only if in every infinite subset of it there is a pair generating a nilpotent subgroup; and Groves [5] showed that this result remains valid when 'nilpotent' is replaced by 'supersoluble'. Spiezia [12] and Longobardi, Mai and Rheumtulla [8] strengthened Neumann's basic hypothesis; and this is used in Edimioni [4] to prove a result having the following as a corollary: a finitely generated soluble group $G$ is nilpotent if, whenever $X, Y$ are infinite subsets of $G$, there exists $x \in X$ and $y \in Y$ so that $\langle x, y\rangle$ is nilpotent. Earlier Lennox [6] had shown the weaker result that a finitely generated soluble group is nilpotent if every two-generator subgroup of it is nilpotent; and subsequently Trabelsi [14] was able to replace Endimioni's 'nilpotent' by 'nilpotent-by-finite'. In [13] Tomkinson showed that, given a positive integer $n$, a finitely generated soluble group has hypercentre of index bounded by a function of $n$ if every subset of cardinality $n$ of the group contains a pair generating a nilpotent subgroup.

Our main result is Theorem 6 given at the beginning of Section 3 . Corollaries of this show that for each of the properties supersolubility, nilpotence and Abelianness there is

Received 4th April, 2006

The author acknowledges helpful comments by Dr L.G. Kovács.

Copyright Clearance Centre, Inc. Serial-fee code: 0004-9727/06 \$A2.00+0.00. 
a function $f$ on the positive integers with the following property. Suppose $G$ is a finite group in which, whenever $X, Y$ are subsets of $G$ of cardinality $n$, there exists $x \in X$ and $y \in Y$ for which $\langle x, y\rangle$ is supersoluble, nilpotent or Abelian: then, whenever $|G|>f(n)$, $G$ is supersoluble, nilpotent or Abelian, as the case may be.

Many articles in the literature build on the work of Lennox and Wiegold [7]; a search for references in this area could usefully start with those given in Trabelsi's articles [14] and [15]. Our notation and terminology is generally that of Doerk and Hawkes [3].

\section{Preliminary Definitions and RESUlts}

For a class $\mathfrak{Y}$ of finite groups and a positive integer $n$ we define the class of finite groups $\mathfrak{Y}^{[n]}$ as follows. A group $G$ is in $\mathfrak{Y}^{[n]}$ if, whenever $X, Y$ are subsets of cardinality $n$ in $G$, there exists $x \in X$ and $y \in Y$ for which $\langle x, y\rangle \in \mathfrak{Y}$. This definition is motivated by that of Spiezia [12] in the context of infinite groups. We adopt the convention that $\mathfrak{Y}^{[n]}$ contains all groups of order less than $n$. Then $\mathfrak{Y}^{[n]}$ is s-closed and is Q-closed whenever $\mathfrak{Y}$ is.

In Theorem 6 we show that, for certain classes $\mathfrak{Y}$ and for all positive integers $n$, $\mathfrak{Y}^{[n]}$ is 'almost' equal to $\mathfrak{Y}$ in the sense that groups in $\mathfrak{Y}^{[\mathfrak{n}]} \backslash \mathfrak{Y}$ have order bounded by a function of $n$.

The classes $\mathfrak{Y}$ we consider are formations with two extra properties. Firstly a finite group $G$ is in $\mathfrak{Y}$ whenever every pair of its elements generates a $\mathfrak{Y}$-group: this is the property Doerk and Hawkes call $\mathfrak{G}_{2}$-completeness, $\mathfrak{G}_{2}$ being the class of 2-generator groups $([3$, p. 516]). Secondly there is a unique Sylow $p$-subgroup in an $\mathfrak{Y}$-group when $p$ is the largest prime dividing its order. That is $\mathfrak{Y}$ is contained in the class $\mathfrak{T}_{>}$of Sylow tower groups with the inverse order on the set of primes ([3, pp. 358-359]). A formation $\mathfrak{Y}$ with these two, extra, properties we shall term a star class. The classes $\mathfrak{A}, \mathfrak{N}$, of finite Abelian and finite nilpotent groups respectively, are star classes, being formations satisfying the extra properties. $U$, the class of finite supersoluble groups, is a star class by a result of Carter, Fischer and Hawkes [2]. (In [2] groups are soluble; however the proof of Corollary 2 below shows that a finite group is necessarily soluble if every pair of its elements generates a supersoluble subgroup.) A proof by induction using another result from [2] - see $\left[3,6.15\right.$ on p. 523] - shows that product classes $\mathfrak{S}_{p_{1}} \mathfrak{S}_{p_{2}} \ldots \mathfrak{S}_{p_{r}}$ are $\mathfrak{G}_{2}$-complete, so are star classes whenever the sequence of primes $\left(p_{i}\right)$ is decreasing. that the minimal simple groups are all two-generator.

In what follows we shall appeal often to the lemmas of this section. Throughout $\phi$ denotes the Euler totient, function and $o(x)$ is the order of the element $x$ of a group.

Lemma 1. Let $\mathfrak{X}$ be a star class, $G$ a group in $\mathfrak{X}^{[n]}$ and $x, y \in G$ with $\phi(o(x)) \geqslant n$. Then $\langle x, y\rangle \in \mathfrak{X}$.

ProOF: There are in $\langle x\rangle$ distinct generators $x_{1}, x_{2}, \ldots, x_{n}$ and the elements 
$x_{i} y(1 \leqslant i \leqslant n)$ are also distinct. Because $G \in \mathfrak{X}^{[n]}$ there are integers $i, j$ for which $\mathfrak{X} \ni\left\langle x_{i}, x_{j} y\right\rangle=\langle x, y\rangle$.

COROLlary 2. Let $\mathfrak{X}$ be a star class and suppose that $G \in \mathfrak{X}^{[n]}$. If $p$ is the largest prime dividing $|G|$ and if $p>n$ then $G$ has unique Sylow $p$-subgroup.

Proof: Let $x, y \in G$ be $p$-elements. Then, since $\phi(o(x)) \geqslant p-1 \geqslant n, H:=\langle x, y\rangle$ $\in \mathfrak{X}$ by Lemma 1 . But $p$ is the largest prime dividing $|H|$ so $x, y \in O_{p}(H)$. Consequently $x y$ is a p-element of $G$. It follows that the set of p-elements of $G$ is a subgroup, so $G$ has a unique Sylow $p$-subgroup.

LEMMA 3. Suppose that $\mathfrak{X}$ is a star class, that $G \in \mathfrak{X}^{[n]}$ and that $N \unlhd G$ with $|N|>n$. Then $G / N \in \mathfrak{X}$.

Proof: Choose elements $g_{1} N, g_{2} N \in G / N$. Since $\left|g_{1} N\right|=\left|g_{2} N\right|>n$ it follows that, for some elements $n_{1}, n_{2} \in N, K:=\left\langle g_{1} n_{1}, g_{2} n_{2}\right\rangle \in \mathfrak{X}$. Hence, in $G / N$,

$$
\left\langle g_{1} N, g_{2} N\right\rangle=K N / N \cong K / K \cap N \in \mathfrak{X}
$$

because $\mathfrak{X}$ is Q-closed. As $\mathfrak{X}$ is a star class, therefore, $G / N \in \mathfrak{X}$.

The next result enables us in Section 3 to reduce to the case of soluble groups; it relies on the classification of finite simple groups.

PROPOS I IION 4. Let $\mathfrak{X}$ be a star class of soluble groups and $n$ a positive integer. The number of isomorphism classes of insoluble groups in $\mathfrak{X}^{[n]}$ is bounded.

Proof: Let $G \in \mathfrak{X}^{[n]} \backslash \mathfrak{S}$, where $\mathfrak{S}$ is the class of finite soluble groups, and suppose that $|G|>n(n !)$. Define $N:=G^{\mathfrak{X}}$ and note that $N \neq\{1\}$ so that there is a chief factor $N / M$ of $G$. It follows from Lemma 3 that $|M| \leqslant n$. Write $C:=C_{G}(M)$. Then, from $|G|=|C| \cdot|G: C| \leqslant|C| \cdot n$ !, we deduce that $|C|>n$. By Lemma 3 again, $G / C \in \mathfrak{X}$ so $N \leqslant C$. In particular $M \leqslant Z(N)$ so $M$ is Abelian. Both $M$ and $G / N$ are soluble so $N / M$ is insoluble. Hence $N / M=S_{1} \times S_{2} \times \cdots \times S_{t}$ where $S_{i} \cong S_{1}(1 \leqslant i \leqslant t)$ and $S_{1}$ is a non-Abelian simple group.

Let $C_{0}:=C_{G}(N / M)$ and note that $C_{0} \cap N=M . G / C_{0}$ is insoluble, so not in $\mathfrak{X}$. Hence, by Lemma $3,\left|C_{0}\right| \leqslant n$. If $t>1$, Lemma 3 shows that $|N / M| \leqslant n^{2}$ so $|G|=\left|C_{0}\right| \cdot\left|G / C_{0}\right| \leqslant n \cdot\left(n^{2}\right)$ !. In the case $t=1$ we invoke Corollary 2 to conclude that all primes dividing $\left|S_{1}\right|$ are less than $n$. By the classification of finite simple groups there is a number $s(n)$ bounding the order of such groups. Then $|G|=\left|C_{0}\right| \cdot\left|G / C_{0}\right| \leqslant n \cdot s(n)$ ! and it follows that

$$
|G| \leqslant \max \left\{n \cdot\left(n^{2}\right) !, n \cdot s(n) !\right\} .
$$

This shows that the orders of insoluble groups in $\mathfrak{X}^{[n]}$ are bounded, establishing the Proposition.

It will be convenient to have an explicit bound for a natural number in terms of its Euler function value. 
LEMMA 5. For every positive integer $m, m \leqslant 2 \phi(m)^{2}$.

ProOF: Suppose $m=\prod_{i=1}^{r} p_{i}^{\alpha_{i}}$ where the $p_{i}$ s are distinct primes in increasing order and with $\alpha_{i} \geqslant 1(1 \leqslant i \leqslant r)$. Then, on the one hand,

$$
\phi(m)=m \prod_{i=1}^{r}\left(1-\frac{1}{p_{i}}\right) \geqslant m\left(1-\frac{1}{2}\right)^{r}=\frac{m}{2^{r}}
$$

so $m \leqslant 2^{r} \phi(m) ;$ and on the other

$$
\phi(m)=\prod_{i=1}^{r} \phi\left(p_{i}^{\alpha_{i}}\right)=\prod_{i=1}^{r} p_{i}^{\alpha_{i}-1}\left(p_{i}-1\right) \geqslant 2^{r-1},
$$

giving $m \leqslant 2 \phi(m)^{2}$.

\section{The MAIN THEOREM}

THEOREM 6. Let $\mathfrak{X}$ be a subgroup closed and saturated star class of groups contained in $\mathfrak{N}$. There is a function $f_{\mathfrak{X}}: \mathbb{N} \rightarrow \mathbb{N}$ for which groups in $\mathfrak{X}^{[n]} \backslash \mathfrak{X}$ have order at most $f_{\mathfrak{X}}(n)$.

The classes $\mathfrak{N}, \mathfrak{U}$, of nilpotent and supersoluble groups respectively, satisfy the hypotheses of this theorem so we have the following corollaries immediately.

COROLlaRY 7. There is a function $f_{\text {nilp }}: \mathbb{N} \rightarrow \mathbb{N}$ for which groups in $\mathfrak{N}^{[n]} \backslash \mathfrak{N}$ have order at most $f_{\text {nilp }}(n)$.

For soluble groups this is consistent with Tomkinson's result [13] in the following sense. Every subset of $2 n$ elements of a group in $\mathfrak{N}^{[n]}$ has a pair that generates a nilpotent subgroup so, by [13], soluble groups in $\mathfrak{N}^{[n]}$ have hypercentre of bounded index; and Corollary 7 ensures this.

COROLlaRY 8. There is a function $f_{s s o l}: \mathbb{N} \rightarrow \mathbb{N}$ for which groups in $\mathfrak{U}^{[n]} \backslash \mathfrak{U}$ have order at most $f_{\text {ssol }}(n)$.

The class $\mathfrak{A}$ is not saturated so does not satisfy the the hypotheses of Theorem 6 . Nevertheless a similar result holds for it.

Corollary 9 . There is a function $f_{a b}: \mathbb{N} \rightarrow \mathbb{N}$ for which groups in $\mathfrak{A}^{[n]} \backslash \mathfrak{A}$ have order at most $f_{a b}(n)$.

We now begin the proof of the Theorem.

Proposition 4 reduces the question to showing that, for each $n \geqslant 1$, soluble groups in $\mathfrak{X}^{[n]} \backslash \mathfrak{X}$ have bounded order. We suppose the theorem to be false and derive a contradiction. That is, we suppose that, for some positive integer $n$, there are groups of arbitrarily large order in $\mathfrak{H}_{n}:=\left(\mathfrak{X}^{[\mathfrak{n}]} \backslash \mathfrak{X}\right) \cap \mathfrak{S}$. 
Our first step is to show that $\mathfrak{H}_{n}$ contains arbitrarily large primitive groups. To this end let $\xi$ be an arbitrary natural number and let $G \in \mathfrak{H}_{n}$ have order at least $n \xi$. Write $N:=G^{\mathfrak{x}}$; it is non-trivial so there is a chief factor $N / M$ of $G$. Now $\mathfrak{X}$ is saturated so $N / M \notin \Phi(G / M)$ and $N / M$ has a complement $C$ in $G$; that is $G=C N, C \cap N=M$. $C$ is a maximal subgroup of $G$ as $N / M$ is an Abelian chief factor of $G$. The factor group $G /$ core $_{G}(C)$ is primitive with a stabiliser $C / \operatorname{core}_{G}(C)$, and kernel (that is, unique minimal normal subgroup) $N$ core $_{G}(C) / \operatorname{core}_{G}(C)$. The latter is the (non-trivial) $\mathfrak{X}$-residual of $G /$ core $_{G}(C)$. By Lemma 3 , $\left|\operatorname{core}_{G}(C)\right| \leqslant n$ so that $\left|G / \operatorname{core}_{G}(C)\right| \geqslant \xi$. That is, writing $\mathfrak{P}_{\mathfrak{n}}$ for the class of primitive groups in $\mathfrak{H}_{\boldsymbol{n}}$ with stabilisers in $\mathfrak{X}$,

there are arbitrarily large groups in $\mathfrak{P}_{n}$.

We observe that $\mathfrak{P}_{n}$ contains groups with arbitrarily large kernels because, if $G=K U$ $\in \mathfrak{P}_{n}$ where $K$ is a stabiliser and $U$ is the kernel, $|K| \leqslant|U|$ !.

Using the same notation suppose that $G=K U \in \mathfrak{P}_{n}$ is a Frobenius group with $K$ generated by two elements, $a, b$ say. If $|U|>2 n$ there are disjoint subsets $U_{1}, U_{2}$ of $U$ of cardinality $n$ and $\left|a^{U_{1}}\right|=\left|b^{U_{2}}\right|=n$. Then, as $G \in \mathfrak{H}_{n}$, for some $u_{1} \in U_{1}$ and $u_{2} \in U_{2}, H_{0}:=\left\langle a^{u_{2}}, b^{u_{2}}\right\rangle \in \mathfrak{X}$. Note that $H_{0} U=G$ so $H_{0} \cap U \unlhd G$. Since $U$ is minimal normal in $G$ it follows that either $H_{0}=G$ or $H_{0} \cap U=\{1\}$. But $G \notin \mathfrak{X}$ so $H_{0} \cap U=\{1\}$. Now $H_{0} \cap K^{u_{1}} \neq\{1\} \neq H_{0} \cap K^{u_{2}}$ so, since $G$ is Frobenius, $K^{u_{1}}=K^{u_{2}}$ thus $u_{1} u_{2}^{-1} \in U \cap N_{G}(K)=\{1\}$ contradicting that $u_{1} \neq u_{2}$. That is $|U|$, and therefore $|G|$, is bounded. In particular groups in $\mathfrak{P}_{n}$ with Abelian, and that is cyclic, stabilisers are Frobenius so have bounded orders.

Now let $\xi$ be arbitrary and suppose that $H:=K U \in \mathfrak{P}_{n}$ where $K$ is a non-Abelian stabiliser, and $U$ the kernel of order greater than $n \xi$. We denote by $p$, a prime, the exponent of $U$; then $O_{p}(K)=\{1\}$ and, since $K$ is nilpotent-by-Abelian, $K^{\prime}$ is a $p^{\prime}$-group. Let $L$ be a minimally non-Abelian subgroup of $K$. Note that $J:=L U \in \mathfrak{H}_{n}$. Since $L^{\prime}$ acts faithfully by conjugation on $U, C_{U}\left(L^{\prime}\right) \leqslant U_{1}<U$ where $U / U_{1}$ is a chief factor of $J$ and $L^{\prime}$ has no non-identity fixed points in $U / U_{1}$ so $\left[L^{\prime}, U\right] U_{1}=U$. We have $J / U_{1} \notin \mathfrak{X}$ or else $J / U_{1} \in \mathfrak{N a}$ which leads to $\left[L^{\prime}, U\right] U_{1}=\left[L^{\prime}, U, L^{\prime}\right] U_{1}=U_{1}$, a contradiction. It follows from Lemma 3 that $\left|U_{1}\right| \leqslant n$ and hence $\left|U / U_{1}\right|>\xi$. Also $L U_{1} / U_{1}$ is maximal in $J / U_{1}$; its core intersects $L^{\prime} U / U_{1}$ trivially; and modulo its core it is minimally non-Abelian. It follows that $J$ has a primitive factor group in $\mathfrak{P}_{n}$ with kernel $U / U_{1}$ and minimally nonAbelian stabilisers. Let $\mathfrak{P}_{n}^{*}$ be the subclass of $\mathfrak{P}_{n}$ of non-Frobenius groups with minimally non-Abelian stabilisers. Since a minimally non-Abelian group is 2-generator the upshot of (1), this paragraph and the last is that

there are groups in $\mathfrak{P}_{\mathfrak{n}}^{*}$ with arbitrarily large kernels.

Our aim now is to show that, on the contrary, the groups in $\mathfrak{P}_{n}^{*}$ do have uniformly bounded orders, thus contradicting our assumption that the theorem is wrong. To this 
end let $G=K U \in \mathfrak{P}_{n}^{*}$ using the usual notation. We first show that

the exponent of $U$ is at most $n$.

Let $p$ be the exponent of $U$. If $p|| K \mid$ then, since $O_{p}(K)=\{1\}, G$ has no normal Sylow $p$-subgroup so, by Corollary $2, p \leqslant n$. If, on the other hand, $p \nmid|K|$, there is a $p^{\prime}$-element $a \in L \backslash\{1\}$ and an element $u \in U \backslash\{1\}$ for which $a u=u a$. Because non-identity central elements of $K$ have no fixed points in $U, a \notin Z(K)$ so, for some $b \in K,\langle a, b\rangle=K$. Then $\langle a u, b\rangle=\langle a, u, b\rangle=G \notin \mathfrak{X}$. Hence, by Lemma 1 ,

$$
n>\phi(o(a u))=\phi(o(a) o(u))=\phi(o(a)) \phi(o(u)) \geqslant \phi(o(u))=p-1
$$

so, in both cases, $p \leqslant n$ as claimed in (3). We note for future reference that much the same argument as in the last sentence, together with Lemma 5 , shows that $o(b) \leqslant 2 n^{2}$.

Suppose that $K$ has a unique maximal normal subgroup, $K_{0}$ say; $\left|K: K_{0}\right|$ is prime since $K$ is soluble. $K$ is not nilpotent because it is not cyclic. Minimality means that $\left|K_{0}\right|$ and $\left|K: K_{0}\right|$ are both prime and each, by Corollary 2 , is at most $n$, so $|K| \leqslant n^{2}$. Now $\mathbb{F}_{p} K$, the regular $K$-module over the field of $p$ elements, contains a section isomorphic to $U$, so $|G| \leqslant p^{n^{2}} \cdot n^{2} \leqslant n^{n^{2}+2}$, using (3).

If, on the other hand, $K$ has different maximal normal subgroups $K_{1}, K_{2}$ then

$$
K=K_{1} K_{2}, K^{\prime}=\left[K_{1}, K_{2}\right] \leqslant K_{1} \cap K_{2} \leqslant Z(K)
$$

and $K$ is nilpotent of class 2 . By minimality $K$ is a $q$-group for some prime $q \neq p$; and $q \leqslant n$ by Corollary 2 . Also $Z(K)$ is cyclic and no non-identity element of it centralises a non-identity element of $U$ as $U$ is faithful and irreducible for the conjugation action of $K$. Because $G$ is not Frobenius there exists $a \in K \backslash\{1\}$ and $u \in U \backslash\{1\}$ for which $a u=u a$; and, as no non-identity power of $a$ is central, we may suppose that $o(a)=q$. As above, for some $b \in K,\langle a, b\rangle$ is not Abelian and therefore it is $K$. However $1=\left[a^{q}, b\right]=[a, b]^{q}$ meaning that $K^{\prime}$ has order $q$. The sentence ending the penultimate paragraph shows that $o(b) \leqslant 2 n^{2}$. Consequently $\left|K / K^{\prime}\right| \leqslant 2 n^{2} \cdot q \leqslant 2 n^{3}$ so $|K| \leqslant 2 n^{3} \cdot q=2 n^{4}$; and then, as in the last paragraph, $|G|$ is functionally bounded.

The last two paragraphs show that there is a uniform bound on the orders of the groups in $\mathfrak{P}_{n}^{*}$. This contradicts (2) and with it the assumption that there are in $\mathfrak{X}^{[n]} \backslash \mathfrak{X}$ groups of unbounded order. The proof of Theorem 6 is therefore complete.

ProOF of Corollary 9: Since $\mathfrak{A} \subseteq \mathfrak{N}$ it follows from Corollary 7 that a group $G$ in $\mathfrak{A}^{[n]}$ of order greater then $f_{\text {nilp }}(n)$ is nilpotent and therefore the direct product of its Sylow subgroups, one of which, a $q$-subgroup $Q$, say, is non-Abelian. Let $N$ be a normal subgroup of $G$ maximal with respect to not containing $Q^{\prime}$. Then $G / N$ is non-Abelian so, by Lemma $3,|N| \leqslant n$. Also $Q^{\prime} N / N$ is the unique minimal normal subgroup of $H:=G / N$ which therefore has class 2 and cyclic centre. Moreover $H \in \mathfrak{A}^{[n]} \backslash \mathfrak{A}$. 
It suffices, therefore, to show that groups $H$ of class 2 and with cyclic centre in $\mathfrak{A}^{[n]}$ have bounded order. Now $H$ is a central product of non-Abelian two-generator groups $H_{i}=\left\langle a_{i}, b_{i}\right\rangle(1 \leqslant i \leqslant m)$ of class 2 with cyclic centre and, possibly, a cyclic group (see [1, Theorem 2.1]). By Lemma 1 an element $h$ with $\phi(o(h)) \geqslant n$ is central so each $H_{i}$ has bounded exponent and therefore bounded order. Moreover with $b:=b_{1} b_{2} \ldots b_{m}$ and $A:=\left\{a_{1}, a_{2}, \ldots, a_{m}\right\},|A|=|A b|=m$ so, if $m \geqslant n$ then, for some $j, k, 1=\left[a_{j}, a_{k} b\right]$ $=\left[a_{j}, b_{j}\right]$ a contradiction. It follows that $H$ is a central product of a group $H_{0}$ of bounded order and a cyclic group $C=\langle c\rangle$. If the result claimed is false then there are such groups $H$ in $\mathfrak{A}^{[n]}$ with arbitrarily large $C$. If $o(c)>o\left(a_{1}\right)$ then $o\left(a_{1} c\right)=o(c)$ so $\phi(o(c))>n$ would mean, by Lemma 1 , that $a_{1} c$, and therefore $a_{1}$, were central, a contradiction. Hence, by Lemma $5,|H|$ is bounded.

\section{Final comments}

Our definition of $\mathfrak{Y}^{[n]}$ is a one-parameter version of a two-parameter definition noted by Neumann [11]. He defines a class we might write as $\mathfrak{Y}^{[m, n]}$ consisting of those groups in which, whenever $X, Y$ are subsets of cardinalities $m, n$ respectively, there is an $x \in X$ and a $y \in Y$ such that $\langle x, y\rangle \in \mathfrak{Y}$.

Direct proofs may be given for Corollaries 7 and 9 independently of our main theorem and not depending on the classification of finite simple groups. By way of example a sketch of a direct proof of Corollary 9 goes like this. If the result is false then there is a smallest $n \geqslant 2$ for which $\mathfrak{A}^{[n]} \backslash \mathfrak{A}$ contains a non-Abelian group $G$ of order greater than $f_{a b}(n-1)$. There are subsets $X, Y$ of cardinality $n-1$ in $G$ for which no $x \in X$ commutes with a $y \in Y$. Observe that $o(x), o(y)$ are bounded by $2 n^{2}$ for $x \in X$ and $y \in Y$ by Lemmas 1 and 5. Either every $g \in G \backslash Y$ commutes with some element of $X$, in which case

$$
G=\bigcup_{y \in Y}\langle y\rangle \cup \bigcup_{x \in X} C_{G}(x)
$$

or, for some $y^{\prime} \in G \backslash Y, y^{\prime}$ commutes with no element of $X$, and then

$$
G=\bigcup_{x \in X}\langle x\rangle \cup \bigcup_{y \in Y} C_{G}(y) \cup C_{G}\left(y^{\prime}\right) .
$$

In the first of these unions not every $\langle y\rangle$, and in the second not every $\langle x\rangle$, is omissible. That is $G$ is an irredundant union of at most $2 n-1$ subgroups whose intersection has order at most $2 n^{2}$. The theorem of Neumann [9] then shows that $|G|$ is bounded by a function of $n$.

A proof of Corollary 7 in this style is somewhat more complicated. 


\section{REFERENCES}

[1] J.M. Brady, R.A. Bryce and J. Cossey, 'On certain abelian-by-nilpotent varieties of groups', Bull. Austral. Math. Soc. 1 (1969), 403-416.

[2] R.W. Carter, B. Fischer and T.O. Hawkes, 'Extreme classes of finite soluble groups', J. Algebra 8 (1968), 285-313.

[3] K. Doerk and T. Hawkes, Finite soluble groups (de Gruyter, Berlin, New York, 1992).

[4] G. Endimioni, 'Groups covered by finitely many nilpotent subgroups', Bull. Austral. Math. Soc. 50 (1994), 459-464.

[5] J.R.J. Groves, 'A conjecture of Lennox and Wiegold concerning supersoluble groups', $J$. Austral. Math. Soc. Ser. 35 (1983), 218-220.

[6] J.C. Lennox, 'Bigenetic properties of finitely generated-(hyper-abelian-by-finite) groups', J. Austral. Math. Soc. 16 (1973), 309-315.

[7] J.C. Lennox and J. Wiegold, 'Extensions of a problem of Paul Erdös on groups', J. Austral. Math. Soc. Ser. A 31 (1981), 459-463.

[8] P. Longobardi, M. Mai and A.H. Rheumtulla, 'Infinite groups in a given variety and Ramsey's theorem', Comm. Algebra 20 (1992), 127-139.

[9] B.H. Neumann, 'Groups covered by finitely many cosets', Publ. Math. Debrecen 3 (1954), 227-242.

[10] B.H. Neumann, 'A problem of Paul Erdös on groups', J. Austral. Math. Soc. Ser. A 21 (1976), 467-472.

[11] B.H. Neumann, 'Ensuring commutativity of finite groups', J. Austral. Math. Soc. 71 (2001), 233-234.

[12] L.S. Spiezia, 'Infinite locally soluble k-Engel groups', Atti Accad. Naz. Lincei Cl. Sci. Fis. Mat. Natur. Rend. Lincei (9) Mat. Appl. 3 (1992), 177-183.

[13] M.J. Tomkinson, 'Hypercentre-by-finite groups', Publ. Math. Debrecen 40 (1992), 313-321.

[14] N. Trabelsi, 'Characterisation of nilpotent-by-finite groups', Bull. Austral. Math. Soc. 61 (2000), 33-38.

[15] N. Trabelsi, 'Soluble groups with many 2-generator torsion-by-nilpotent subgroups', $P u b l$. Math. Debrecen 67 (2005), 93-102.

Mathematical Sciences Institute

The Australian National University

Canberra, ACT 0200

Australia 